\title{
Adhesive assisted fabrication of chirped POF Bragg grating
}

Min, Rui, Pereira, Luís, Paixão, Tiago, Woyessa, Getinet, André, Paulo, et al.

Rui Min, Luís Pereira, Tiago Paixão, Getinet Woyessa, Paulo André, Ole Bang, Anselmo Frizera, Paulo Antunes, Joao Pinto, Zhaohui Li, Beatriz

Ortega, Carlos Marques, "Adhesive assisted fabrication of chirped POF Bragg grating," Proc. SPIE 11355, Micro-Structured and Specialty Optical Fibres VI, 1135510 (1 April 2020); doi: 10.1117/12.2555432

SPIE. Event: SPIE Photonics Europe, 2020, Online Only 


\title{
Adhesive assisted fabrication of Chirped POF Bragg grating
}

\author{
Rui Min ${ }^{\mathrm{a}, \mathrm{b}}$, Luis Pereira ${ }^{\mathrm{c}}$, Tiago Paixão ${ }^{\mathrm{c}}$, Getinet Woyessa ${ }^{\mathrm{d}}$, Paulo André ${ }^{\mathrm{e}}$, Ole Bang ${ }^{\mathrm{d}}$, Anselmo \\ Frizera $^{\mathrm{f}}$, Paulo Antunes ${ }^{\mathrm{c}}$, Joao Pinto ${ }^{\mathrm{c}}$, Zhaohui Li ${ }^{\mathrm{g}}$, Beatriz Ortega ${ }^{\mathrm{b}}$, and Carlos Marques ${ }^{\mathrm{c}}$ \\ ${ }^{a}$ School of Intelligent Systems Science and Engineering, Jinan University, Zhuhai 519070, China \\ bITEAM Research Institute, Universitat Politècnica de València, 46022 Valencia, Spain \\ ${ }^{c}$ I3N and Physics Department, Universidade de Aveiro, 3810-193 Aveiro, Portugal \\ ${ }^{d}$ DTU Fotonik, Department of Photonics Engineering, Technical University of Denmark, Denmark \\ ${ }^{\mathrm{e}}$ Instituto Superior Técnico - Lisbon University and Instituto de Telecomunicações, Lisbon, Portugal \\ ${ }^{\mathrm{f}}$ Graduate Program in Electrical Engineering, Federal University of Espirito Santo, Vitória, Brazil \\ ${ }^{g}$ State Key Laboratory of Optoelectronic Materials and Technologies and School of Electronics and \\ Information Technology, Sun Yat-sen University, 510275 Guangzhou, China
}

\begin{abstract}
FBG in polymer optical fibers (POFs) is a promising technology for a wide range of sensing applications due to a lower Young's modulus and a large range of applying strain. Furthermore, POFs have several properties which make them attractive for biosensing applications such as non-brittle nature, flexibility in bending and biocompatibility. Chirped Fiber Bragg gratings (CFBGs), which are characterized by a non-uniform modulation of the refractive index show a broad reflection spectrum, enabling short-length distributed sensing. The combining benefits of POF and CFBGs is attractive for biomedical applications. Here, we present a novel method to obtain CFBG in POF with a post-process uniform POF FBG by using resin.
\end{abstract}

Keywords: Fiber Bragg grating, polymer optical fiber, laser irradiation, adhesive.

\section{INTRODUCTION}

Fiber Bragg grating sensors are emerging as a promising technology among the broad field of fiber optics sensors [1]. Since the first demonstration of the photo-induced modulation of the refractive index in the 90s [2], and theoretical work that analyzes grating structures in fiber employing layer peeling [3] and coupled-mode theory [4], FBG sensors have attracted huge interest due to its inherent advantages, such as lightweight, immunity to electromagnetic fields, fast response, suitability for the harsh environment, etc [5].

FBG sensors show emerging applications in healthcare and medical devices [6], structural engineering [7], oil and gas monitoring applications [8-10]. Uniform FBGs are based on the periodical modulation of the refractive index of the fiber core, and they are the most popular grating-based device. Non-uniform grating has enriched the type of FBG, which depends on the type of refractive index modulation and fiber characteristics. By modulating the refractive index amplitude, it is possible to generate apodized gratings, which has a higher rejection of spectral side lobes. Recently, tilted FBGs with tilting grating profile to excite cladding modes also attracted attention for biomedical applications with high precision [1112].

All these types of gratings have a spatially uniform periodicity. By varying the period of the refractive index modulation along the grating, it is possible to obtain a chirped FBG (CFBG) [13]. In a CFBG, the Bragg wavelength varies along the grating, because each portion of the grating reflects a specific wavelength according to the Bragg's condition. As a result, the CFBG has a reflection spectrum which is much broader than uniform FBGs, with a range from a few nanometers to tens of nanometers in $3 \mathrm{~dB}$ bandwidth.

CFBG has been widely studied and employed in optical communications, as they could provide compensation for the group delay due to long haul optical transmission [14]. Recently, CFBG also gained interest from the sensing community thanks to the key features and the inherent grating structure [15]. The most significantly key characteristic of the CFBG is the fact that all spectrum depends not only on the temperature or strain applied on the whole grating length but on the 
information recorded in each section of the gratings. From this point, the use of a CFBG sensor can potentially detect rates of spatial changes of strain or temperature.

Polymer optical fiber (POF) has several advantages over silica fiber, such as a larger elongation before breakage, a lower Young's modulus, and a higher thermo-optic coefficient. These advantages will provide a broad tuning range by either heating or straining the fiber. Apart from these, biocompatibility is an important feature for bio-medical applications. The fabrication technology of grating devices in silica fiber is mature. However, the fabrication technology for grating devices in POF is still under research [16-20], with the first CFBG in polymer optical fiber obtained by Marques et al with the phase mask method in 2017 [21]. Then, tapering, gradient annealing and femtosecond direct writing method [22-24] were investigated to obtain CFBG in POF for different application scenarios [25-28]. In this paper, to the best of our knowledge, we report a novel method to fabricate CFBG in POF with adhesive resin.

\section{CFBG WORKING PRINCIPLE}

The working principle of a CFBG extends from the uniform grating structure as is shown in Fig. 1. For a uniform FBG, the Bragg wavelength is equal to [4]:

$$
\lambda_{B}=2 \Lambda n_{e f f},
$$

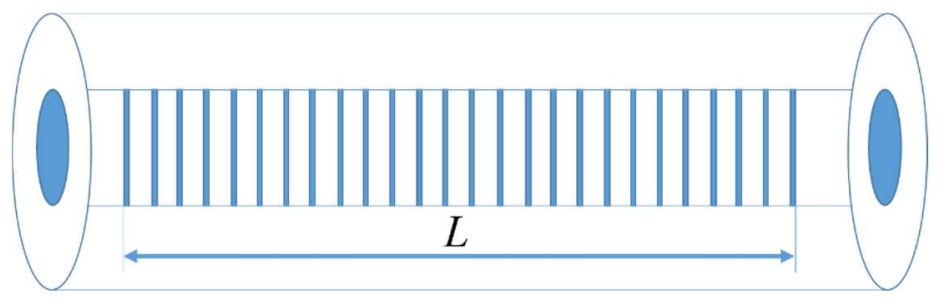

Fig. 1. Uniform grating structure.

where $\Lambda$ the period of the refractive index modulation, $\mathrm{n}_{\mathrm{eff}}$ is the effective refractive index of the fiber core and $\mathrm{L}$ is the length of the grating. Moreover, for a chirped FBG, the periodicity of the modulation is not constant as shown in Fig. 2. It changes along the propagation axis z; the function $\Lambda(\mathrm{z})$ defines the chirp pattern. This implies that each section of the grating reflects a different Bragg wavelength, and the total spectrum of the FBG consists on the sum of the reflected wavelengths from each section of the grating.

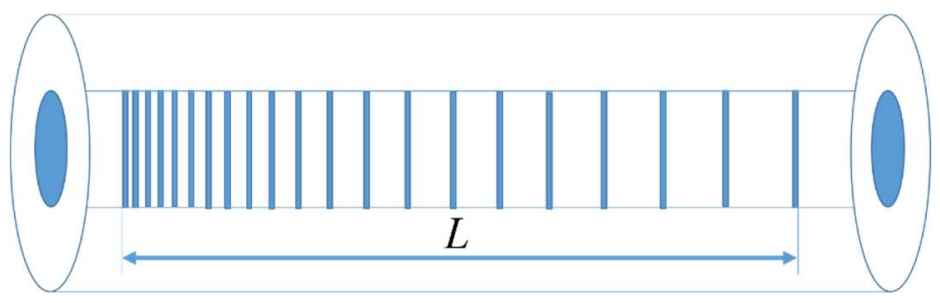

Fig. 2. Chirped Bragg grating structure.

In a linear CFBG, as displayed in Fig. 2, the chirp rate coefficient is constant and defines the rate of spatial change of the Bragg wavelength within the grating structure. The parameters of the refractive index modulation (effective refractive index of the fiber core, $n_{\text {eff, }}$ amplitude about modulation of the refractive index, $\delta n_{\text {eff }}$, coupling coefficient, $k$ ) are assumed to be constant over the whole grating length. The reference value for the Bragg wavelength has a linear dependence upon the grating length. 


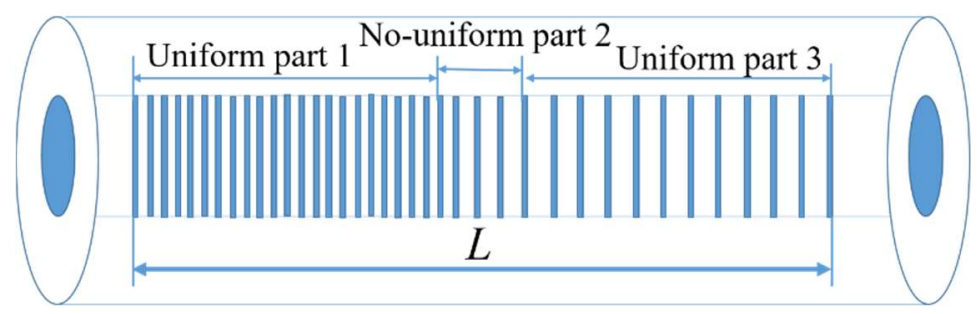

Fig. 3. Special CFBG structure.

For a special Bragg grating as presented in Fig. 3, the grating consists of one uniform grating with a narrow period, one linear CFBG and a uniform grating with broad period grating. The reflect spectrum also shows chirp performance which could be calculated with the coupled-mode theory [4]. Actually, in silica fiber there is no signature requirement to achieve such kind of grating due to the fabrication process and the limited applications. However, in POF, which has a better elastic performance, it is possible to obtain such structure by post-processing an uniform FBG in POF, by stretching the fiber and gluing part of the grating. We will demonstrate this procedure in the next section of this work.

\section{POF CFBG FABRICATION}

The fiber samples used in this work were undoped 2-ring PMMA microstructured POF (mPOF) with an average hole diameter and a pitch in the fiber of $1.70 \mu \mathrm{m}$ and $3.95 \mu \mathrm{m}$, respectively (fabricated in DTU Fotonik [29]). The samples were pre-annealed at $70^{\circ} \mathrm{C}$ for 12 hours to remove any residual stresses created during the drawing process. Then, the fiber samples were cleaved with a homemade portable polymer optical fiber cleaver [30] and connected with typical ferrule to simplify the interrogation of the reflected optical signal. Fig. 4 shows the cross-section of the fiber.

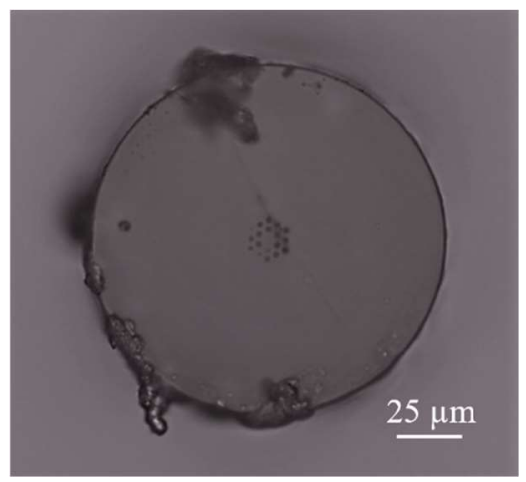

Fig. 4. Cross-section of the 2-ring PMMA mPOF.

In our fabrication setup, a pulsed Q-switched Nd:YAG laser system (LOTIS TII LS-2137U ) lasing at the fourth harmonic operating at $266 \mathrm{~nm}$ wavelength was employed for the uniform FBG inscription with a pump lamp repetition rate of $5 \mathrm{~Hz}$ as is displayed in Fig. 5. The laser beam profile is circular, the diameter is about $8 \mathrm{~mm}$ and the divergence is $\leqslant 1.0 \mathrm{mrad}$. The laser beam is focused onto the fiber core using a plano-convex cylindrical lens. The effective spot size of the beam on the fiber surface is $8 \mathrm{~mm}$ in width and about $30 \mu \mathrm{m}$ in height. The phase mask is $10 \mathrm{~mm}$ length with a period of $\Lambda_{\mathrm{PM}}=$ $567.8 \mathrm{~nm}$, which is designed to operate at $248 \mathrm{~nm}$ wavelength. 


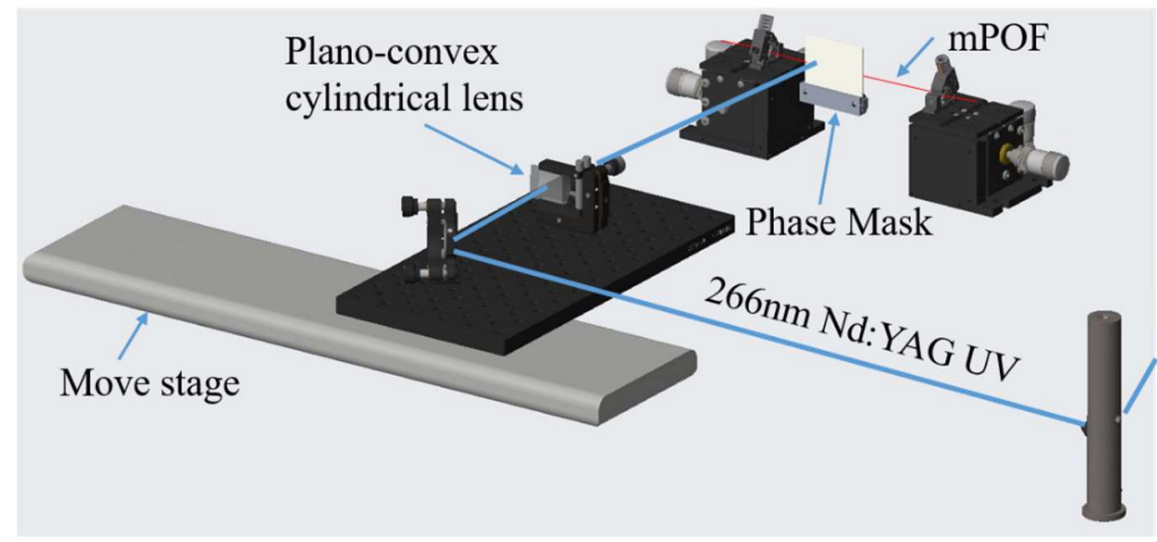

Fig. 5. Schematic of the laser irradiation system.

The FBG reflected optical power was monitored during irradiation by employing a superluminescent diode (Superlum SLD-371-HP1) and an optical spectrum analyzer (Yokogawa AQ6373B) with $0.02 \mathrm{~nm}$ resolution. We observed a Bragg grating device with $28 \mathrm{~dB}$ of reflectivity using $5 \mathrm{~Hz}$ of frequency during 180 seconds after spectrum stabilization as shown in Fig. 6.

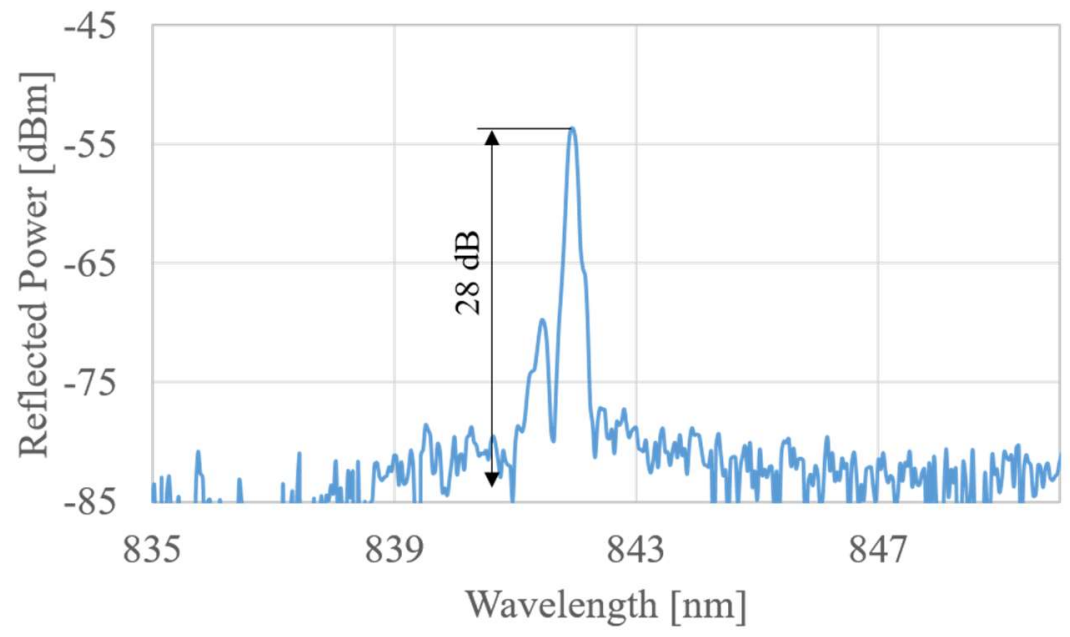

Fig. 6. Reflected spectral power of the FBG.

Norland optical adhesive, a solvent-free material which could be fully cured in few minutes by ultraviolet light exposure, was used to glue half part of the grating ( $4 \mathrm{~mm}$ of the grating, since the total of grating length was $8 \mathrm{~mm}$ ). It was applied strain $(\sim 1 \%)$ in the Bragg grating and then the curing process started with the Norland 86 coating on half of the grating length. As expected, the reflected power spectrum became broader during UV exposure as shown in Fig. 7, which indicates a stretch performance appears due to the coated half of the grating under UV exposure. After releasing the fiber, due to the combined performance of resin and release, all spectrum blue shifted, and became narrower than the previous one. Both chirped reflected power spectra show some apodized performance due to liquid coating during the UV curing process, which makes it difficult to obtain an uniform coating on the fiber. The interface between the coated and uncoated parts of the grating show some slope on the coating thickness, which implies a non-uniform strain performance. 


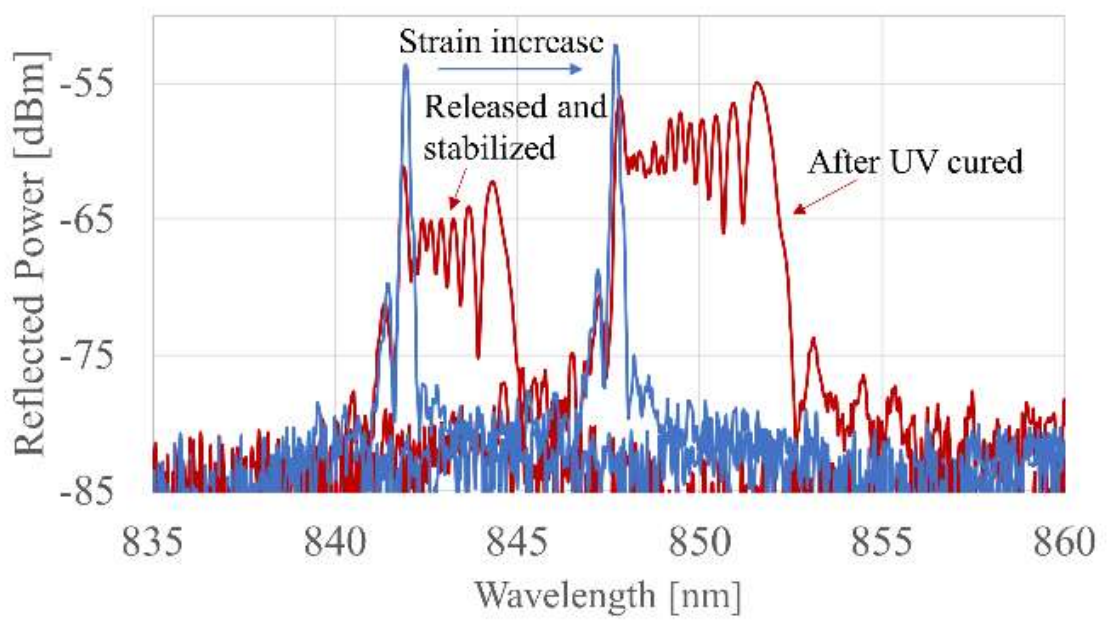

Fig.7. Grating reflected spectra before (blue) and after (red) applying resin under $1 \%$ strain, and spectrum of the grating after released and stabilized.

Once the curing process was finished, the fiber was released and stabilized for several days. The measured reflected power spectrum shows a bandwidth reduction down to $\sim 3 \mathrm{~nm}$, as shown in Fig. 7.

\section{CONCLUSION}

As conclusion, we present the working principle of a novel CFBG production technique in POF, by obtaining this kind of device for the first time, which is a simple post-process method to achieve CFBG, based on the large elastic performance of POF. Future research will focus on the characterization of the device and applications in biomedical field.

\section{ACKNOWLEDGMENT}

The Spanish Ministerio de Ciencia, Innovación y Universidades RTI2018-101658-B-I00 FOCAL Project; National Natural Science Foundation of China (61435006, 61525502); The Science and Technology Planning Project of Guangdong Province (2017B010123005, 2018B010114002); Local Innovation and Research Teams Project of Guangdong Pearl River Talents Program (2017BT01X121). This work was developed within the scope of the project i3N, UIDB/50025/2020 \& UIDP/50025/2020, financed by national funds through the FCT/MEC. Luís Pereira and Tiago Paixão acknowledge FCT for the grants SFRH/BD/146295/2019 and PD/BD/128265/2016, respectively. This work is also funded by national funds $(\mathrm{OE})$, through FCT-I.P., in the scope of the framework contract foreseen in the numbers 4,5 and 6 of the article 23, of the Decree-Law 57/2016, of August 29, changed by Law 57/2017, of July 19.

\section{REFERENCES}

[1] Rao, Y. J., "In-fibre Bragg grating sensors,"Measurement Science and Technology 8(4), 355(1997).

[2] Meltz, G., Morey, W., \& Glenn, W. H., "Formation of Bragg gratings in optical fibers by a transverse holographic method," Optics Letters 14(15), 823-825 (1989).

[3] Skaar, J., Wang, L., \& Erdogan, T., "On the synthesis of fiber Bragg gratings by layer peeling," IEEE Journal of Quantum Electronics 37(2), 165-173(2001).

[4] Erdogan, T., "Fiber grating spectra," Journal of Lightwave Technology 15(8), 1277-1294(1997).

[5] Othonos, A. S., \& Kalli, K., "Fiber Bragg gratings fundamentals and applications in telecommunications and sensing," Artech house, (1999).

[6] Liu, Z., \& Tam, H. Y., "Industrial and medical applications of fiber Bragg gratings," Chinese Optics Letters 14(12), 120007(2016). 
[7] Antunes, P., Varum, H., \& Andréa, P., "Optical FBG sensors for static structural health monitoring," Procedia Engineering 14, 1564-1571(2011).

[8] Qiao, X., Shao, Z., Bao, W., \& Rong, Q., "Fiber Bragg grating sensors for the oil industry," MDPI Sensors 17(3), 429(2017).

[9] Rong, Q., \& Qiao, X., "FBG for oil and gas exploration," Journal of Lightwave Technology 37(11), 25022515(2018).

[10]Zhou, X., Dai, Y., Zou, M., Karanja, J. M., \& Yang, M., "FBG hydrogen sensor based on spiral microstructure ablated by femtosecond laser," Sensors and Actuators B: Chemical 236, 392-398(2016).

[11] Guo, T., Tam, H. Y., Krug, P. A., \& Albert, J., "Reflective tilted fiber Bragg grating refractometer based on strong cladding to core recoupling," Optics Express 17(7), 5736-5742 (2009).

[12] Albert, J., Shao, L. Y., \& Caucheteur, C., "Tilted fiber Bragg grating sensors," Laser \& Photonics Reviews, 7(1), 83-108(2013).

[13] Tosi, D., "Review of chirped fiber bragg grating (CFBG) fiber-optic sensors and their applications," MDPI Sensors 18(7), 2147(2018).

[14] Capmany, J., Pastor, D., \& Ortega, B., "New and flexible fiber-optic delay-line filters using chirped Bragg gratings and laser arrays," IEEE Transactions on Microwave Theory and Techniques 47(7), 1321-1326(1999).

[15] Tosi, D., Macchi, E. G., Gallati, M., Braschi, G., Cigada, A., Rossi, S., Leen G. \& Lewis, E., "Fiber-optic chirped FBG for distributed thermal monitoring of ex-vivo radiofrequency ablation of liver," Biomedical Optics Express 5(6), 1799-1811(2014).

[16] Webb, D. J., "Fibre Bragg grating sensors in polymer optical fibres," Measurement Science and Technology 26(9), 092004 (2015).

[17]Luo, Y., Yan, B., Zhang, Q., Peng, G. D., Wen, J., \& Zhang, J., "Fabrication of polymer optical fibre (POF) gratings," MDPI Sensors 17(3), 511(2017).

[18] Broadway, C., Min, R., Leal-Junior, A. G., Marques, C., \& Caucheteur, C., "Toward commercial polymer fiber Bragg grating sensors: Review and applications," Journal of Lightwave Technology 37(11), 2605-2615(2019).

[19] Min, R., Ortega, B., \& Marques, C., "Latest Achievements in Polymer Optical Fiber Gratings: Fabrication and Applications," MDPI Photonics 6(2), 36 (2019).

[20] Theodosiou, A., \& Kalli, K., "Recent trends and advances of fibre Bragg grating sensors in CYTOP polymer optical fibres," Optical Fiber Technology 54, 102079(2020).

[21] Marques, C. A. F., Antunes, P., Mergo, P., Webb, D. J., \& André, P., "Chirped Bragg gratings in PMMA stepindex polymer optical fiber," IEEE Photonics Technology Letters 29(6), 500-503(2017).

[22] Min, R., Ortega, B., \& Marques, C., "Fabrication of tunable chirped mPOF Bragg gratings using a uniform phase mask," Optics Express 26(4), 4411-4420(2018).

[23] Min, R., Ortega, B., Broadway, C., Caucheteur, C., Woyessa, G., Bang, O., Antunes P, \& Marques, C., "Hot water-assisted fabrication of chirped polymer optical fiber Bragg gratings," Optics Express 26(26), 34655-34664 (2018).

[24] Theodosiou, A., Hu, X., Caucheteur, C., \& Kalli, K.,"Bragg gratings and Fabry-Perot cavities in low-loss multimode CYTOP polymer fiber," IEEE Photonics Technology Letters 30(9), 857-860(2018).

[25] Min, R., Ortega, B., Broadway, C., Hu, X., Caucheteur, C., Bang, O., Antunes P. \& Marques, C.,"Microstructured PMMA POF chirped Bragg gratings for strain sensing," Optical Fiber Technology, 45, 330-335 (2018).

[26] Min, R., Korganbayev, S., Molardi, C., Broadway, C., Hu, X., Caucheteur, C., Bang O., Antunes P., Tosi D., Marques C. \& Ortega, B. ," Largely tunable dispersion chirped polymer FBG," Optics Letters, 43(20), 5106-5109 (2018).

[27] Korganbayev, S., Min, R., Jelbuldina, M., Hu, X., Caucheteur, C., Bang, O., Ortega B., Marques C. \& Tosi, D.,"Thermal profile detection through high-sensitivity fiber optic chirped Bragg grating on microstructured PMMA fiber,"Journal of Lightwave Technology 36(20), 4723-4729 (2018).

[28] Leal, A. G., Rocha H. R. O., Theodosiou A., Frizera A., Marques C., Kalli K., Ribeiro Moisés R. N., " Optimizing Linearity and Sensitivity of 3D-Printed Diaphragms with Chirped FBGs in CYTOP Fibers, "IEEE Access in press (2020).

[29] Min, R., Pereira, L., Paixão, T., Woyessa, G., André, P., Bang, O. Antunes P., Pinto J., Li Z.H., Ortega B. \& Marques, C., "Inscription of Bragg gratings in undoped PMMA mPOF with Nd: YAG laser at $266 \mathrm{~nm}$ wavelength, " Optics Express, 27(26), 38039-38048(2019).

[30] Sáez-Rodríguez, D., Min, R., Ortega, B., Nielsen, K., \& Webb, D. J., "Passive and portable polymer optical fiber cleaver, " IEEE Photonics Technology Letters 28(24), 2834-2837(2016). 